\title{
Knowledge-Based System Guided Automatic Contour Segmentation of Abdominal Structures in CT Scans
}

\author{
Mahmoud Saleh Jawarneh, Mohammed Said Abual-Rub \\ Computer Science Department, Al-Imam Muhammad Ibn Saud Islamic University, Ahsa, Saudi Arabia \\ Email address: \\ gawrnah@imamu.edu.sa (M. S. Jawarneh), msabualrub@imamu.edu.sa (M. S. Abual-Rub)
}

\section{To cite this article:}

Mahmoud Saleh Jawarneh, Mohammed Said Abual-Rub. Knowledge-Based System Guided Automatic Contour Segmentation of Abdominal Structures in CT Scans. International Journal of Intelligent Information Systems. Vol. 5, No. 1, 2016, pp. 5-16. doi: $10.11648 /$ j.ijiis. 20160501.12

\begin{abstract}
The advice of automating computer applications is being increase to reduce the human interaction. Medical image segmentation is one of these applications, when done manually; it turns into a time-consuming and knowledge intensive task. As a result, automatic segmentation is in the focus of work to speed up segmentation processes. Fast and accurate segmentation would allow physicians to analyze and visualize the human structures and re-plan radiation therapy and surgery. This paper introduces a knowledge system based on different sources of medical knowledge to automate medical image segmentation through active contour methods. The way of getting benefit of the knowledge provided by medical atlas, expert's rules, image features, image multiple views and image Meta data introduced by this knowledge system. We classify the system in different domains in way can be manage properly to guide active contour segmentation methods for abdominal CT scans. The obtained results are very promising showing significant improvements over other methods where the volume measurements error is $7 \%$ and the processing time was improved by $68 \%$.
\end{abstract}

Keywords: Knowledge-Based System, Medical Knowledge, Active Contour Image Segmentation, Automated Processes

\section{Introduction}

Image segmentation can be identified as the process of isolating different regions in an image having homogenous features such as intensity, texture, color etc. Image segmentation is considered as an essential and crucial preliminary processing analysis and one of the most challenging tasks in any computer vision systems (Gonzalez and Woods, 2008). Image segmentation plays a major role in various imaging fields. Medical imaging is one of the mostly referred fields by the imaging community (Zhou, Yang et al. 2014).

Medical imaging produces datasets that may require considerable amount of processing time for one particular human anatomy. A typical Magnetic Resonance Imaging (MRI) and Computed Tomography (CT) scan results in large number of slices of the acquired anatomical region. This stack of 2D images are referred as dataset; these images appear in gray scale color with each gray scale value corresponding to an Hounsfield Units (HU), which is a measure of radiodensity that provides an accurate absolute density for the corresponding anatomical tissues (Möller 2009).
A typical processing of medical images includes preprocessing to remove noise and segmentation process that delineate a particular anatomy of interest. The highly complex nature of medical images makes segmentation difficult and time consuming. It also may require sophisticated segmentation algorithms to obtain reliable results.

Image segmentation plays a crucial role in medical imaging application, includes the classification of different anatomies such as bone, soft tissues and muscles; visualization of medical image; volumetric measurement; shape representation and analysis; computer guided surgery; treatments planning and human anatomy changes detection. Besides, there has been a growing need for segmentation in anatomical structure studies in research and teaching (O'Donnell 2001; Withey and Koles 2007; Cui, Wang et al. 2013).

Depending upon the case studies, medical image segmentation can be a very complicated, tedious and time consuming. Moreover, manual delineation is a highly skilled, subjective and laborious task. Selecting each pixel manually for desired the anatomical structure in every medical slice of a dataset that may consists of more than 50 slices could take hours or even days (Casiraghi, Campadelli et al. 2009; Zhou 
and Xie 2014). As the processing resources of the computers have seen some advancement, the automation of medical image segmentation can be performed with higher accuracy, repeatability, and efficiency (O'Donnell 2001; Straka, Cruz et al. 2004; ChangYang, Xiuying et al. 2010; Luo, Li et al. 2014). Fast and accurate segmentation would allow physicians to analyze and visualize human anatomies and assist radiation therapy and surgical planning.

This paper is organized as follows. Section 2 explains the aim of our work in comparison with other works in medical knowledge used in medical image segmentation. Section 3 introduces the knowledge system architecture that represents the different domains of knowledge. Section 4 describes the methodology and processes flow used to guide the medical image segmentation. Section 5 discusses the results of proposed work. And Section 6 describes the conclusion of this work.

\section{Related Work}

Many different image segmentation methods have been developed in the past several decades in medical imaging domain. However, image segmentation remains acutely problem centric. A given segmentation method may perform well on one problem but poorly on a different application. Thus, achieving a generic segmentation method that is universally applicable for a broad range of medical applications is a very challenging task. The variety of medical applications encourages research interests on the segmentation process in order to develop, improvised and advanced methods for a given application.

Existing segmentation methods in medical domain include neural network learning methods which require training dataset to build the constraints such as intensity, texture, shape etc, that need to be given into neural network (ChienCheng et al., 2003; (Yasmin, Sharif et al. 2013)); intensitybased methods that are based on similarity in intensity and needs initialization value (Campadelli et al., 2009); rulebased recognition based on exploiting structure invariants and available features such as size, edge and location (ChienCheng and Pau-Choo 2000; Narkhede 2013); model-based methods that need training sets to build a model to guide the segmentation process (Heimann et al., 2007; (Jiang, Tan et al. 2013)); active contour methods that require initialization of contour inside target structure (Lee et al., 2007; (Zhou and Xie 2014)); atlas-based segmentation which requires registration between atlas and target dataset (Furukawa et al., 2007; (Yongfu, Tianzi et al. 2012)) and finally unsupervised methods such as clustering techniques produces clusters belonging to many different structures. It normally combines with other methods in order to isolate specific structure (Yuqian, Yunlong et al. 2010).

Active contour segmentation methods (Pan and Dawant 2001; Liu, Jiang et al. 2005; Furukawa, Shimizu et al. 2007; Lee, Kim et al. 2007; Martí, Benedí et al. 2007; Zhou and Xie 2014) have specific advantages over other methods, such as providing promising results, robustness to dataset variations, no prior training, and ability to capture the topology of shapes (Li, Fevens et al. 2006). As such, they can be reliably used for segmenting structures in abdominal CT scans. However, active contour methods have some disadvantages, that is longer processing time due to the need of user interaction, to plot the contour of level set in each slice in the abdominal dataset, which is very time consuming and knowledge intensive task. In addition, performance of level set active contour methods relies heavily on having a good initialization of the contour curve close to the desired contour.

Depending on user interaction and prior knowledge, medical image segmentation algorithms can be classified as manual, semi-automatic and automatic. Automatic methods still need prior knowledge such as shape, location and texture relating to the human anatomy to be segmented. In addition some of these methods also require initialization. As examples, active contour segmentation methods needs curve initialization; region growing segmentation methods need seed point initialization, etc (Foo 2006; Mei, Si et al. 2013). Therefore, medical knowledge represented by variety of sources such as medical atlas, texture information, anatomical shape and location, is necessary for image processing, especially for image segmentation.

Survey indicates that there are huge demands for automating the segmentation of abdominal structures. It includes measurement of kidney volumes, which is a good pointer of common body parameters and a reliable predictor of renal function (Shin, Chung et al. 2009); measurement of liver volume, which is useful for liver transplantation (Nakayama, Li et al. 2006; Luo, Li et al. 2014); constructing volume of abdominal structures helpful in surgical planning and radiation treatment (Harms, Bartels et al. 2005; Luo, Li et al. 2014). CT scans are preferred more than sensitive imaging techniques such as MRI in abdominal imaging owing to their high signal to noise and good spatial resolution (Linguraru, Sandberg et al. 2010). But it is noted that abdominal images segmentation is complex and challenging task due to several reasons contributed by high similarities in the gray levels among different structures, the surrounding soft tissues as well as inhomogeneity in shape and texture of the same structure in different image slices (Ding, Leow et al. 2005).

This paper addresses the challenges in improving the level of automation and reducing processing time while improving the accuracy of the segmentation. This paper concentrates on adapting the medical knowledge to automate the segmentation of abdominal structures (liver, spleen, left kidney and right kidney) in CT scan using active contour segmentation methods.

Establishing an explicit framework that models the relation among various sources of medical knowledge is a necessity. It will facilitate integration and reuse of valuable medical knowledge in guiding segmentation techniques. Furthermore, it will serve as an automated system for segmenting medical images that will in turn help the medical practitioners enormously. This paper proposes a framework called the 
knowledge-based segmentation framework to address this need.

The proposed segmentation system is generic, and employs multiple sources of medical knowledge: medical atlas; expert's rules; image features; multiple view CT dataset (axial, coronal and sagittal) and DICOM image Meta data. This proposed segmentation system introduces several ideas to overcome the challenges of segmenting abdominal structures automatically. These challenges are: localizing the desired abdominal structure, initializing and propagating the contour curve in all abdominal structure slices, localizing discontinuity regions in liver and initializing contour curve on them.

\section{Knowledge Representation}

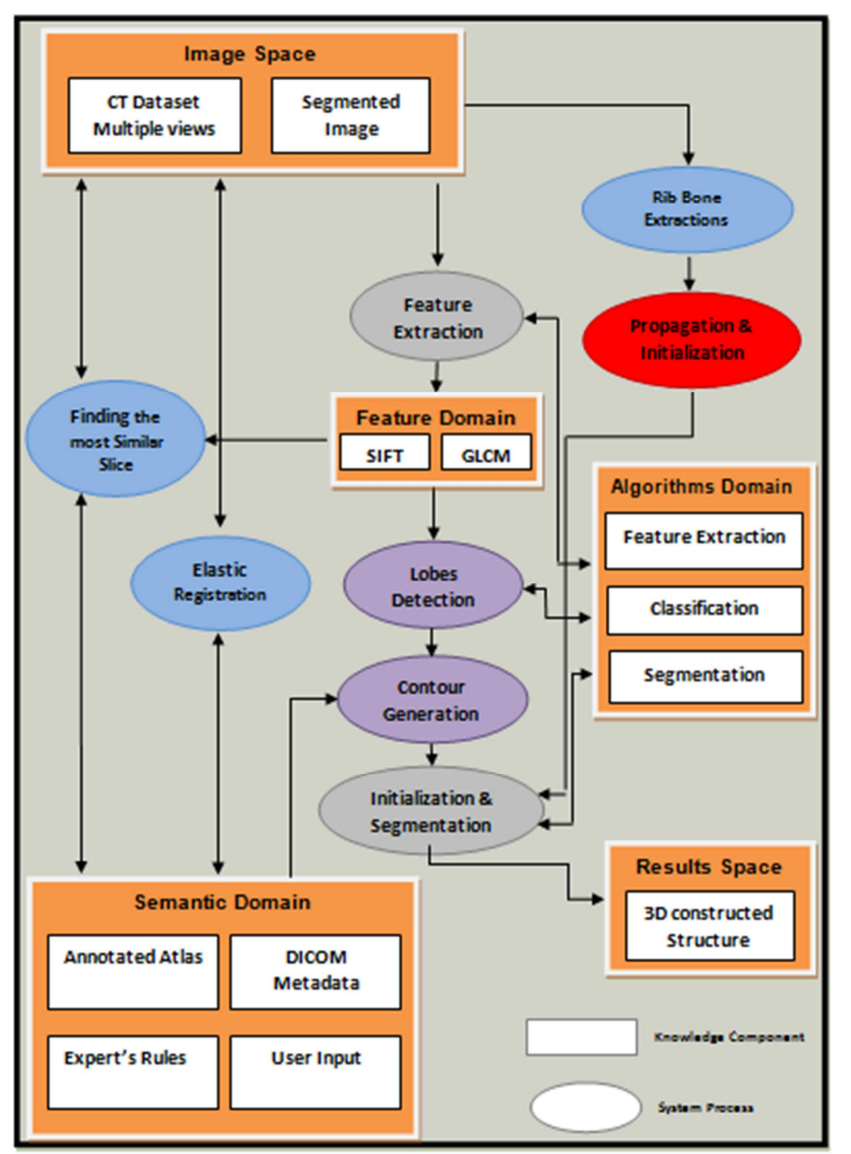

Figure 1. Knowledge-based framework of the proposed segmentation system.

The architecture of the proposed system shown in Figure 1 consists of five knowledge components. The semantic domain consists of multiple sources of knowledge namely: medical atlas, experts' rules, user information and DICOM metadata. Image space contains input CT dataset slices and segmented image during system processing. The Features domain contains extracted features from the input image, segmented image and medical atlas image. The algorithm domain contains feature extraction algorithms, classification algorithms and active contour segmentation algorithms.
Finally result space to save the final segmentation results of the segmented structure. Also, the system consists of nine system processes which are: finding the most similar slice, elastic registration, rib bones extractions, initialization, propagation and initialization, feature extraction, multiple lobes localization, contours generation and segmentation. The arrows in Figure 1 show the interaction between knowledge components that are represented in rectangles and knowledge processes that are represented in oval shapes. The following section describes these components.

\subsection{Knowledge Components}

The proposed system consists of five knowledge components namely, image space, algorithm domain, feature domain, semantic domain and result space.

\subsubsection{Semantic Domain}

This domain represents the semantic feature (high level feature) of human anatomy from several knowledge sources listed below:

- Medical atlas: A medical atlas is a representation of anatomical information primarily through annotated images and is intended to serve radiologists and other physicians. The primary concern for this paper is an annotated atlas which consists of two files: the annotated slice for abdominal region and the XML file. There are many available annotated medical atlases that can be used such as the radiology anatomy atlas (Hancock and Kittler 1990; RadiologyAnatomyAtlas 2010). Figure 2 shows an example atlas image taken from the Radiology anatomy atlas.

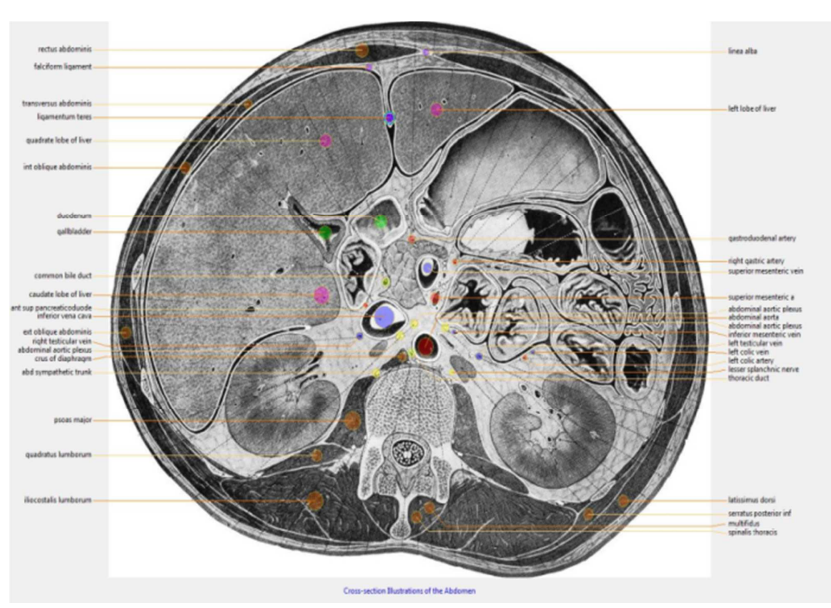

Figure 2. Radiology anatomy atlas. (RadiologyAnatomyAtlas 2010).

There are also many available software that can be used to generate simple annotated atlas images such as AnnoteImage software (SIG 2010). Figure 3 shows a snapshot of annotating image by AnnoteImage software.

An annotated image is an image that is described by words representing a brief description of the image contents. The words are assigned to image contents using arrows. The word descriptions and arrow locations are saved as a metadata file in a machine readable XML format associated with the image 
file (Jia and Wang 2008). In this paper a typical image is chosen as the atlas image from one of the available CT datasets and is annotated using AnnoteImage software (SIG 2010). Figure 4 shows an example of the annotated image.

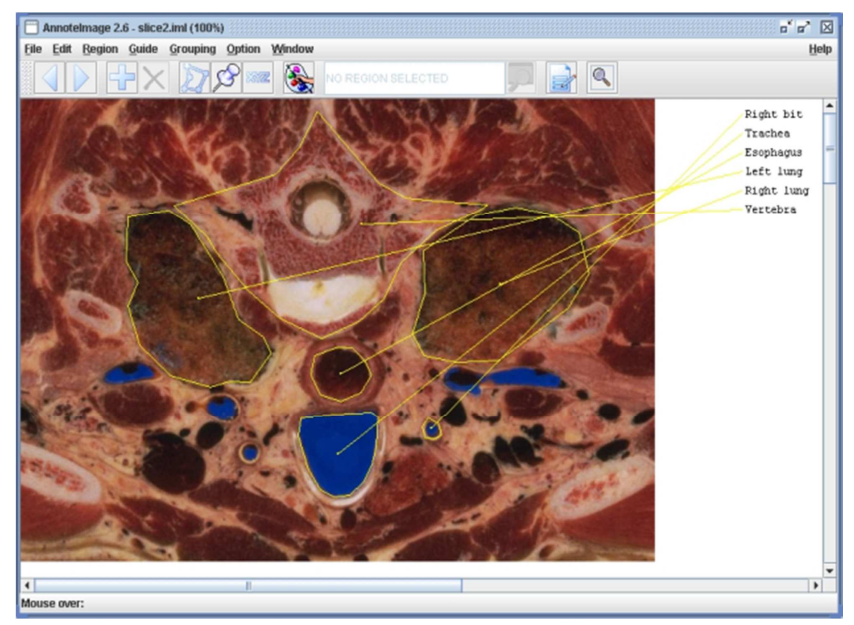

Figure 3. AnnoteImage software. (SIG 2010).

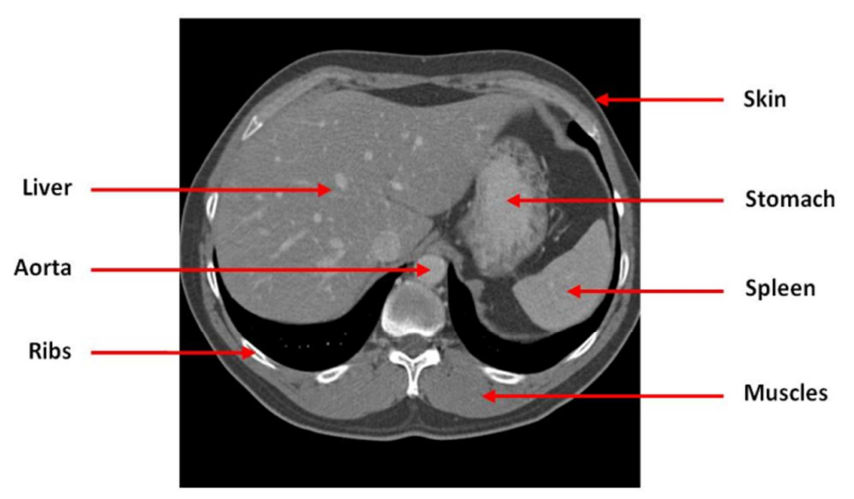

Figure 4. Annotated atlas image.

- Medical experts' rules: These are representations of medical expert knowledge related to the abdominal anatomies in the human body. Experts' rules are necessary to guide the segmentation of abdominal structures. This may help in narrowing down the search space and sustaining in reduced processing time. In addition it probably improves the performance as a result of the injection of additional knowledge into the process. For each target anatomy, a set of rules is used taking into account recommendations from experts as well as low level image observations. Some examples of the rules or previous anatomical knowledge about the liver that the system can get benefitted from are: liver structure is located inside the rib bones; liver lobes are mainly located in the right part of the abdomen; liver is a continuous 3D object etc.

- DICOM metadata: Digital Imaging and Communications in Medicine (DICOM) is a standard for handling, storing, printing, and transmitting information in medical imaging. A DICOM image contains modality image and DICOM metadata. DICOM metadata consists of image header parameters such as patient name, age, modality information, image attributes such as slice thickness, slice spacing, pixel spacing and resolution (rows and columns) (Kallman, Halsius et al. 2008). The proposed system makes use of the slice spacing and the pixel resolution (rows, columns) information to guide the active contour segmentation methods for the abdominal structures.

- User input: it will be the selection of target structure in input dataset based on its name and it is the only user interaction in this system.

\subsubsection{Image Space}

Image space is the data storage (database) of datasets that need to be segmented to get specific abdominal human anatomy. Input datasets provide the system with important knowledge which can be used in guided segmentation through multiple views of abdominal CT dataset (axial, sagittal and coronal) as in Figure 5. The intersection between these views can be used as an important knowledge source in guiding active contour segmentation. Coronal or sagittal view of the abdominal anatomy helps to define the starting and ending axial slice of the target structure. Image space also contains a segmented image which temporarily stores the result of the processing operations in the input image such as registration, initialization, propagation and segmentation processes.

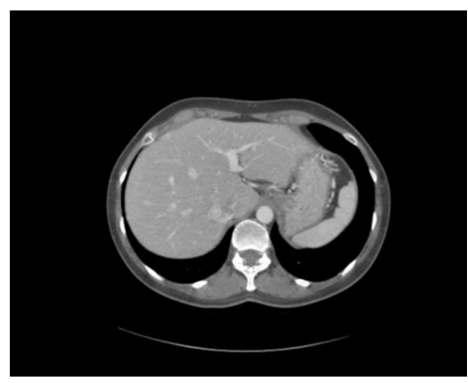

(a)

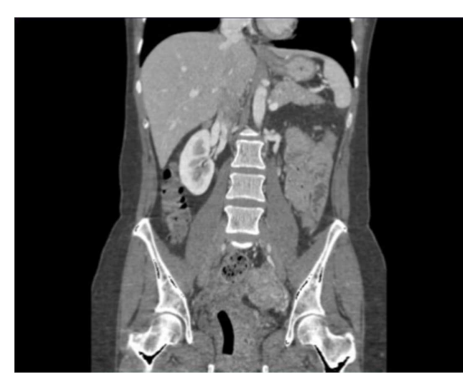

(b)

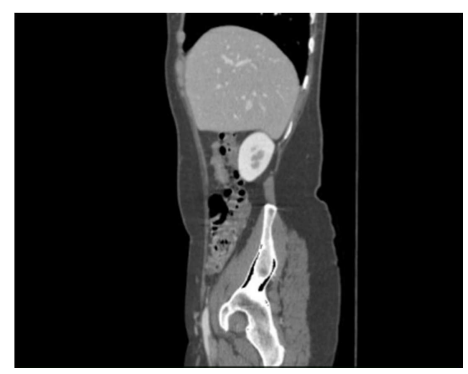

(c) 


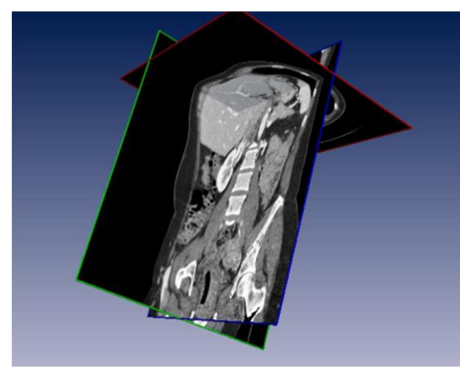

(d)

Figure 5. (a) Axial; (b) Coronal; (c) Sagittal; (d) All; views of abdominal dataset.

\subsubsection{Algorithm Domain}

The algorithm domain in the proposed system contains various algorithms used in this system. These algorithms can be classified into three categories. The first category comprises of active contour segmentation algorithms used in the medical domain such as (Chan and Vese 2001; Pan and Dawant 2001; Chunming, Chenyang et al. 2005; Lee, Kim et al. 2007; Lankton and Tannenbaum 2008). The second category contains feature extraction algorithms: Haralick texture features, gray level co-occurrence matrix (GLCM) (Haralick 1973) and scale-invariant feature transform (SIFT) (Lowe 2004). The third category includes classification algorithms: Principal Component Analysis (PCA) implemented in (Kroon, Oort et al. 2008).

\subsubsection{Feature Domain}

Temporary data storage is used to store the extracted features of abdominal structures from CT dataset slices and segmented images as well as the extracted features of abdominal anatomies in the medical atlas image. This system uses two types of texture features to guide the active contour segmentation algorithms. The first type comprises Haralick texture features (GLCM) (Haralick 1973) used with PCA classifier to detect multi lobes in some abdominal structures like the liver. The second type contains scale-invariant feature transform (SIFT) (Lowe 2004) used to find the most similar slice in $\mathrm{CT}$ abdominal dataset with the annotated atlas slice.

\subsubsection{Result Space}

The final output of this system is the $3 \mathrm{D}$ construction of abdominal structure in all 2D slices in CT dataset saved in result space.

\subsection{System Processes}

The system processes are the processes used in the proposed knowledge-based system:

A. Segmentation: This process uses the active contour segmentation method to isolate target abdominal structure in CT dataset.

B. Feature extraction: A process used to extract the texture features from CT dataset slices, segmented images and atlas image. These features (SIFT and GLCM) are used in this system as a knowledge source to guide the active contour method.
C. Finding most similar slice: A process used to find the most similar slice in CT dataset to the annotated atlas 2D image. In other words, it can be called automatic structure localization.

D. Elastic registration: This is a process to assist in transferring an initial point within the anatomy of interest from the atlas image to the same anatomical region within the target slice.

E. Rib bone extractions: A process to extract rib bones and remove muscle tissues between those rib bones to avoid the similarity in intensity between abdominal structure and the muscles during the active contour segmentation.

F. Initialization: Process used to initialize the initial contour curve of active contour method inside the target anatomy in the most similar slice to atlas image based on the location of initial point $(\mathrm{x}, \mathrm{y})$ taken from XML file of the atlas image.

G. Propagation and initialization: A process used to automatically propagate the initialization of the contour curve of the active contour segmentation method to all CT dataset slices belonging to the target anatomy. The multiple CT dataset views axial, sagittal and coronal are used as a source of 3D shape knowledge anatomy in this process.

H. Lobes localization: This process utilizes GLCM texture features of specific abdominal anatomy that has multiple lobes to locate the regions of these lobes with the assistance of expert rules.

I. Contour generation: A process which generates the initial contour curve inside the detected lobe region.

The system can be extended to segment additional anatomical structures by adding a new knowledge source such as new annotated atlases, build new experts rules, semantic networks etc. Also, the system's architecture allows other algorithms "plugged-in" to the system such as another active contour method "plugged-in" to the segmentation algorithm domain, another classification method "pluggedin" to classification algorithm domain etc. In addition, new feature can be used in the features domain. With appropriate extensions, the knowledge framework can be extended to other modalities.

\section{Methodology}

As an initial step, the system builds the static knowledge source references (which can be used for any CT dataset) namely: the annotated atlas image, experts' rules. Medical annotated atlas images represent the abdominal anatomies in the abdominal part of the human body. The atlas images is built as follows: For each abdominal anatomy one slice is chosen from normal CT dataset which approximately corresponds to the largest cross-section, which is usually the middle slice with regard to the upper most and lower most slices of the abdominal structure. In choosing this slice it is ensured that the selected anatomy is contained in a single connected region. The chosen anatomy is annotated by pointing to its mid position. The study uses available 
software such as AnnoteImage software (SIG 2010) for the annotation task. The Annotated atlas image consists of the image and an XML file that contains the name of the anatomy and the annotated points.

Experts' rules are a set of rules developed by recommendations from experts in radiology. Normally, the radiologist performs manual segmentation of the anatomy based on their anatomical facts. The system uses these facts as a set of rules to guide the automatic active contour segmentation. The rules that are adapted in the proposed system for liver anatomy are based on previous anatomical knowledge, such as: "liver structure located inside the rib bones", "liver lobes are mainly located in the right part of the abdomen", "Liver is a continuous 3D object". The rules for spleen structure are: "spleen is located inside the rib bones", "in the left part of the abdomen" and "it is a continuous 3D object". The rules for kidney structure are: "kidney occupies two parts one located in the right side of the abdomen and the other in the left side", and "it is a continuous 3D object".

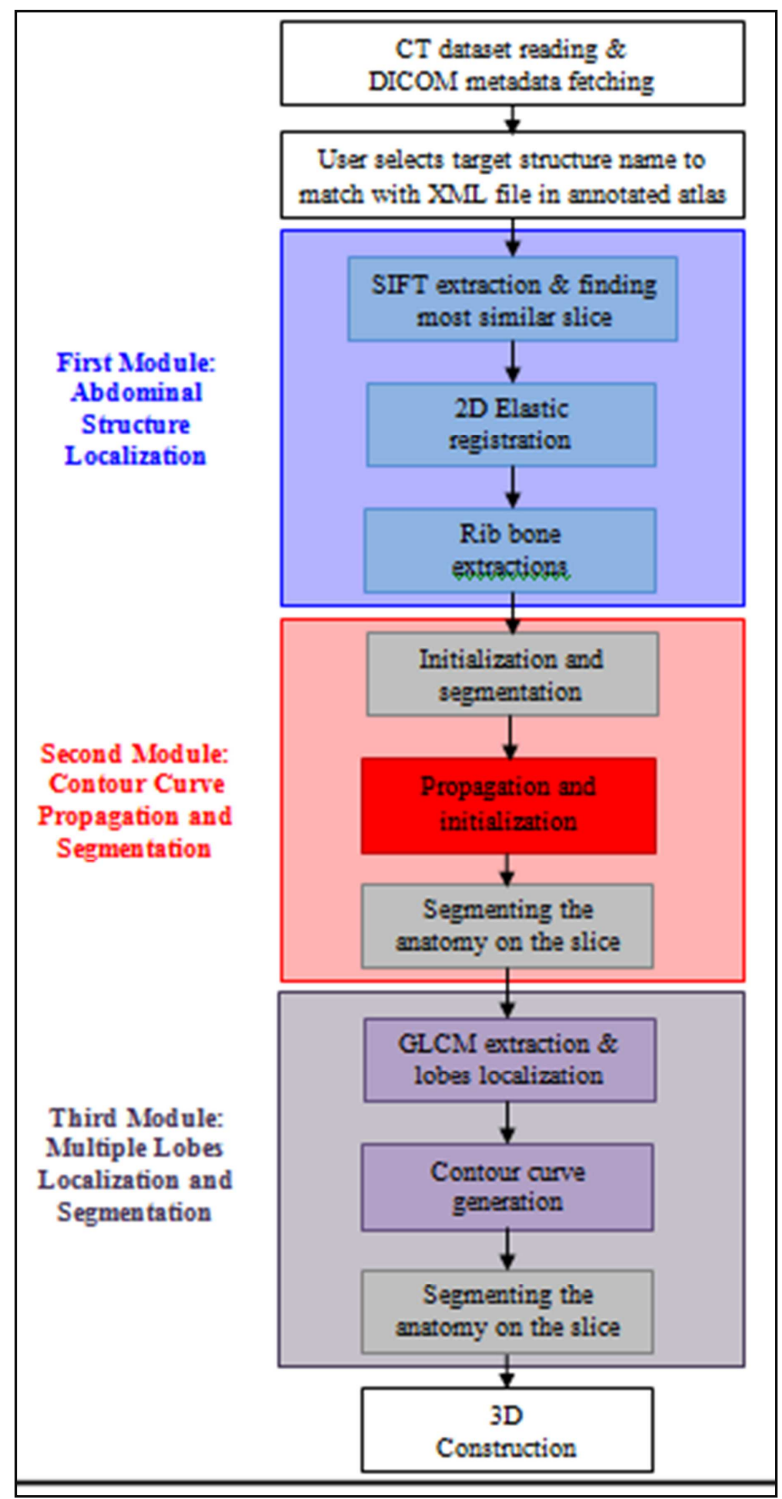

Figure 6. The proposed system flow.
The algorithm domain consider as a source of knowledge of the used algorithms in the system. Texture features algorithms which are SIFT and GLCM, classification algorithm is PCA and segmentation algorithm is active contour segmentation. Other source of knowledge that may be considered as a temporary source is for every new dataset CT scan has its own multiple views (axial, coronal and sagittal) and its own DICOM metadata information.

The work flow of the system is shown in Figure 6. As has been mentioned earlier, this knowledge system is suitable for abdominal CT scan datasets. When the system reads the new dataset, it fetches the meta-data (DICOM header file) to get the necessary information used by it. After that the user selects the name of the target abdominal structure from a list of structure names. The system automatically chooses the suitable annotated atlas image related to the selected anatomy. For example, if the user selects the liver structure, the system will choose the annotated atlas image for liver. Next the system matches the name of the selected anatomy and the names of anatomies in the annotated atlas image through XML file to get $(\mathrm{x}, \mathrm{y})$ location for the target anatomy. The proposed system is divided to three main modules: Abdominal structure localization, contour curve propagation and multiple lobes detection.

\section{Results and Discussions}

Most authors in the literature employed their own private datasets to evaluate their methods. An objective comparison among different segmentation methods would not be accurate and fair, due to the shortage of a common dataset with its ground truth, and a unique performance criterion between the segmentation methods and the manual segmentation of each abdominal structure (Campadelli, Casiraghi et al. 2009; Abdel-massieh, Hadhoud et al. 2010). Eight abdominal structures (five livers, one spleen, one right kidney and one left kidney) are extracted in this paper from six CT datasets, more details in the following subsections.

\subsection{Abdominal Datasets}

One of the main focuses of this paper is to segment the liver structure automatically from abdominal CT datasets. In a typical CT scan of the abdomen in axial view, liver starts as a single object and grows until reaching a maximum size, then gets smaller, divides into separate lobes and finally disappears. It is worth mentioning that the liver is considered the most difficult human anatomy to be segmented automatically (Ibrahim, Petrou et al. 2010).

This paper uses a 3D ground truth for the datasets provided for liver, namely, the "MICCAI2007 grand challengeSegmentation of the Liver" workshop (MICCAI 2007). This grand challenge was established to compare between liver segmentation methods as $3 \mathrm{D}$ segmented result. The evaluation criteria used in this challenge is suitable for $3 \mathrm{D}$ segmentation.

The proposed system tested on five contrasts enhanced CT datasets in the database of the liver segmentation grand 
challenge (http://sliver07.isi.uu.nl/, accessed: October 2010). These datasets have pixel resolution varying between 0.55 and $0.8 \mathrm{~mm}$; inter slice distance ranging from 1 to $3 \mathrm{~mm}$, with each axial slice having 512 X 512 pixels. The datasets in DICOM format with gray level in the range $[-1024,+3071]$, are related to the Hounsfield units (HU). Gold standard is created by experts through an interactive tool by using the intensity-based seeded region grower followed by drawing freehand boundaries in case of inaccurate delineation. The available datasets used from this challenge namely Liver1, Liver3, Liver4, Liver5 and Liver6; each dataset consists of
183, 79, 212, 319 and 111slices respectively.

For other abdominal structures, the spleen, right kidney and left kidney, there is no available ground truth. The proposed system tested on the contrast enhanced CT dataset in the database of the Osirix medical software (http://www.osirix-viewer.com/, accessed: October 2010). Each axial slice has 512 X 512 pixels in this dataset. This dataset was used to extract spleen, left kidney and right kidney while Gold standard is created through an interactive tool, this dataset consists of 120 slices. Table 1 provides information about abdominal structures datasets.

Table 1. Abdominal structures datasets.

\begin{tabular}{lllll}
\hline Abdominal structure name & Abdominal dataset name & Dataset source & Number of slices & Range of abdominal structure slices \\
\hline liver & Liver1 & MICCAI2007 & 183 & $62-163$ \\
liver & Liver3 & MICCAI2007 & 79 & $14-70$ \\
liver & Liver4 & MICCAI2007 & 212 & $57-196$ \\
liver & Liver5 & MICCAI2007 & 319 & $110-272$ \\
liver & Liver6 & MICCAI2007 & 111 & $20-92$ \\
spleen & Spleen & Osirix & 120 & $46-118$ \\
right kidney & Right Kidney & Osirix & 120 & $7-86$ \\
left kidney & Left Kidney & Osirix & 120 & $20-104$ \\
\hline
\end{tabular}

\subsection{Evaluation of Constructed 3D Volume}

$3 \mathrm{D}$ volume is constructed from $2 \mathrm{D}$ segmentation results for each abdominal structure. The evaluation of this $3 \mathrm{D}$ constructed volume is evaluated using quantitative measures for $3 \mathrm{D}$ segmentation. In this work, we use six metrics for evaluating $3 \mathrm{D}$ segmentation results. Of these, five metrics are those suggested in the MICCAI2007 liver challenge namely volumetric overlap error, relative volume difference, average symmetric surface distance, symmetric root mean square (RMS) surface distance and Maximum symmetric surface distance (Ginneken, Heimann et al. 2007). The sixth is the criterion volume measurement error (Nakayama, Li et al. 2006). In the following explanation of these criterions, the segmentation result is termed AS and the gold standard is termed GT. S(AS) and S(GT) represent the set of surface voxels of $A$ and $B$ respectively:

1) Volumetric overlap error (VOE).

Volumetric overlap represents the number of voxels in the intersection of the 3D segmentation result and the 3D gold standard, divided by the number of voxels in the union of the $3 \mathrm{D}$ segmentation result and the 3D gold standard. This criterion is similar to Jaccard coefficient in 2D where the value of volumetric overlap is subtracted from 1 to get the volumetric overlap error as in equation (1). This is a perfect segmentation if this value is equal to 0 . This value is equal to 1 if there is no overlap at all between the segmentation results and the gold standard. The value is multiplied by 100 to represent it in percent.

$$
V O E=100\left[1-\frac{|A S \cap G T|}{|A S \cup G T|}\right]
$$

2) Relative volume difference (RVD).

The total volume of the $3 \mathrm{D}$ segmentation is divided by the total volume of the gold standard. From this number, 1 is subtracted, the absolute value is taken, and the result is multiplied by 100 as in equation (2). The value is given in millimeters; the perfect segmentation is 0 and larger than zero otherwise. Note that the perfect value of 0 can also be obtained for a non-perfect segmentation as long as the volume of that segmentation is equal to the volume of the gold standard.

$$
R V D=100\left[\frac{|A S|}{|G T|}-1\right]
$$

3) Average symmetric surface distance (ASSD).

The border voxels of the 3D segmentation result and the 3D gold standard are determined. These are defined as those voxels in the objects that have at least one neighbour (from the 26 nearest neighbours) that does not belong to the object. For each voxel in these sets, the closest voxel in which set is determined (using Euclidean distance and real world distances, taking into account the generally different resolutions in the different scan directions). All these distances are stored for border voxels from both 3D gold standard and 3D segmentation result. The average of all these distances gives the average symmetric absolute surface distance. The value is given in millimeters and the perfect segmentation is 0 .

$$
d(v, S(A S))=\min _{S A S \in S(A S)}\|v-s A S\|
$$

Where $\|$.$\| denotes the Euclidean distance. The Average$ symmetric surface distance (ASSD) is obtained using equation (4).

$$
A S S D(A S, G T)=\frac{1}{|S(A S)|+|S(G T)|}\left(\sum_{S A S \in S(A S)} d(s A S, S(G T))+\sum_{S G T \in S(G T)} d(s G T, S(A S))\right)
$$

Symmetric root means square surface distance (SRMSSD). 
This measure is similar to the previous measure, but stores the squared distances between the two sets of border voxels. After averaging the squared values, the root is extracted and the SRMSSD is obtained as in equation (5). This value is 0 for a perfect segmentation.

$$
\operatorname{SRMSSD}(A S, G T)=\sqrt{\frac{1}{|S(A S)|+|S(G T)|}} \times \sqrt{\left(\sum_{S A S \in S(A S)} d^{2}(S A S, S(G T))+\sum_{S G T \in S(G T)} d^{2}(s G T, S(A S))\right)}
$$

4) Maximum symmetric surface distance (MSSD).

This measure is similar to the previous two, but only the maximum of all voxel distances is taken instead of the average as in equation (6). The value is given in millimeters and the perfect segmentation is 0 .

$$
\operatorname{MSSD}(A S, G T)=\max \left\{\max _{S A S \in S(A S)} d(s A S, S(G T)), \max _{s G T \in S(G T)} d(s G T, S(A S))\right\}
$$

5) Volume measurement error

This measure is used to compare both volumes: automatic segmented volume and manual segmented volume. To obtain the structure volume, the structure segmented area in each slice is calculated by counting the number of pixels in this area and then having it multiplied by pixel spacing. These areas are summed up and multiplied by slice thickness yielding the total volume. Then, volume measurement error is calculated based on equation (7).

$$
\text { Volume measurement error }=\left(\frac{\text { Volume }_{\text {automatic }}}{\text { Volume }_{\text {manual }}}-1\right) \times 100 \%
$$

The scoring system suggested in MICCAI2007 challenge is used to evaluate the proposed system. The reference values given by an independent user observer that yield scores of 75 for the liver segmentation are shown in Table 2. Using this scoring system, the score more than or equal to 75 points for a segmented liver are considered equivalent to performance by the manual segmentation (Ginneken, Heimann et al. 2007). Therefore, a score below 75 points may still considered very good (Ginneken, Heimann et al. 2007).

Table 2. Reference values for scoring system of liver segmentation (MICCAI 2007).

\begin{tabular}{lll}
\hline Performance criterion & Value & Score \\
\hline Volumetric overlap error (VOE) [\%] & 6.4 & 75 \\
Relative volume difference (RVD) [\%] & 4.7 & 75 \\
Average symmetric surface distance (ASSD) [mm] & 1.0 & 75 \\
Symmetric root mean square surface distance & 1.8 & 75 \\
(SRMSSD) [mm] & 19 & 75 \\
Maximum symmetric surface distance (MSSD) [mm] & 19 & \\
\hline
\end{tabular}

The results of segmenting the five Liver datasets by the proposed system are compared with the results obtained using other methods established in MICCAI2007 challenge. These results are presented in Figure 7, Tables 3, 4 and 5. Note that the comparison is limited to methods proposed in (Tibamoso and Rueda 2007) and (Slagmolen, Elen et al. 2007) as no published results are available for the other methods.

The method in (Tibamoso and Rueda 2007) is considered to be a semi-automatic segmentation method that isolate the $3 \mathrm{D}$ liver volume from CT datasets. It is based on shape modeling followed by discrete deformable surfaces. A shape model of the liver is formulated and manually initialized in the target liver dataset as an initial surface for 3D deformable surface function. The $3 \mathrm{D}$ deformable surface is guided by intensity and edges attributes. In the semi-automatic method proposed in (Slagmolen, Elen et al. 2007), 20 training liver datasets, segmented manually, are used to build an atlas via non-rigid registration. Then, non-rigid registration is utilized again to register the atlas with the volume of interest in target dataset. As show in table 3 the segmentation score by the proposed system is $62 \%$. The score registered by (Tibamoso and Rueda 2007) method is $69 \%$ as in table 4 . The score registered by (Slagmolen, Elen et al. 2007) method is 59\% as in table 5. From this, it may conclude that the proposed system is comparable to other methods by taking into account that the proposed work is an automatic whereas the other methods are semi-automatic methods. The proposed also system does not make explicit use of training data. Furthermore, as been mentioned earlier the performance of the proposed system is not only depends upon the proposed method but also on the level set active contour algorithm being utilized. In other word, a more efficient active contour

\begin{tabular}{|c|c|c|c|c|c|c|}
\hline Dataset Name & VOE [\%] & RVD [\%] & ASSD [mm] & SRMSSD [mm] & MSSD [mm] & Total Score \\
\hline Liver1 & 9 & 5.5 & 1.8 & 3.6 & 40.4 & 57.6 \\
\hline Liver3 & 7.2 & 4.1 & 1.2 & 3.3 & 22.2 & 68.8 \\
\hline Liver4 & 8.8 & 2.6 & 1.8 & 3.5 & 34.9 & 61.6 \\
\hline Liver5 & 7.7 & 6.2 & 1.1 & 3.7 & 26.4 & 65.2 \\
\hline Liver6 & 8.7 & 6.5 & 2 & 3.8 & 26.4 & 57.8 \\
\hline Average & & & & & & 62 \\
\hline
\end{tabular}
algorithm may exhibit better segmentation results.

Table 3. 3D performance measurements for liver segmentation by the proposed system. 
Table 4. 3D performance measurements for liver segmentation by (Tibamoso and Rueda 2007).

\begin{tabular}{lllllll}
\hline Dataset Name & VOE [\%] & RVD [\%] & ASSD [mm] & SRMSSD [mm] & MSSD [mm] & Total Score \\
\hline Liver1 & 10.7 & 5.8 & 2 & 4.5 & 33.4 \\
Liver3 & 6.2 & 3.8 & 1 & 0.5 & 2.8 & 22.1 \\
Liver4 & 4.3 & 2.8 & 0.9 & 2.1 & 12.5 \\
Liver5 & 7.8 & 6 & 1.4 & 3.9 & 85 \\
Liver6 & 7.8 & 4.2 & & & 34 \\
Average & & & & 62 & \\
\hline
\end{tabular}

Table 5. 3D performance measurements for liver segmentation by (Slagmolen, Elen et al. 2007).

\begin{tabular}{|c|c|c|c|c|c|c|}
\hline Dataset Name & VOE [\%] & RVD [\%] & ASSD [mm] & SRMSSD [mm] & MSSD [mm] & Total Score \\
\hline Liver1 & 9.71 & 1.53 & 1.84 & 3.69 & 35.5 & 61 \\
\hline Liver3 & 5.68 & 1.04 & 1 & 2 & 24.23 & 77 \\
\hline Liver4 & 8.41 & 6.24 & 1.82 & 5.18 & 70.08 & 47 \\
\hline Liver6 & 10.44 & 8.05 & 2.26 & 5.01 & 44.79 & 47 \\
\hline Average & & & & & & 59 \\
\hline
\end{tabular}

Table 6. Volume measurements of automatic and manual segmentations.

\begin{tabular}{llll}
\hline Dataset Name & Volume Of Automatic Segmentation [mI] & Volume Of Manual Segmentation [mI] & Volume Measurement Error (\%) \\
\hline Liver1 & 2527.3 & 2386.4 & 5.9 \\
Liver3 & 1346.4 & 1212.2 & 11.1 \\
Liver4 & 3020.9 & 2944.4 & 2.6 \\
Liver5 & 3919.6 & 3613.6 & 8.4 \\
Liver6 & 1505.1 & 1374.7 & 9.4 \\
Spleen & 329 & 304 & 8 \\
Left Kidney & 293 & 276 & 6 \\
Right Kidney & 245 & 233 & 5 \\
Average & & & 7.0 \\
\hline
\end{tabular}

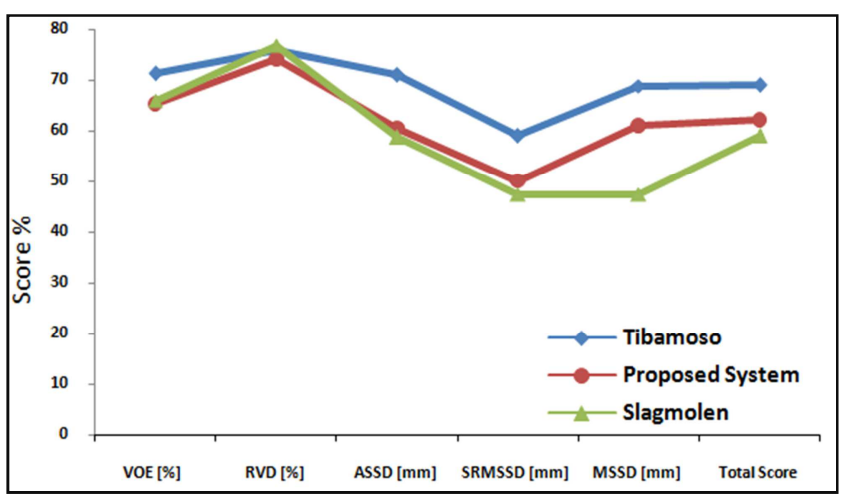

Figure 7. Comparison of $3 D$ performance results.

The sixth criterion used to evaluate $3 \mathrm{D}$ results is the volume measurements error. Comparisons of volume measurements error provided by manual and automatic segmentations for five liver datasets, spleen, left kidney and right kidney are shown in Table 6 with an average error of $7.0 \%$.

\subsection{Performance of Processing Time}

The farther initialization of contour curve is from final desired boundary, the more computation time is required for contour curve to reach final position. Hence, if the contour curve starts in the exact place which is near to the abdominal structure boundary, the time needed for segmentation can be drastically reduced. Thus, utilization of knowledge, especially on the location of abdominal structures plays an important role in the context of this work.

One of the major strength of the proposed system is the speed up of the segmentation process. The processing time is referred to as the time required completing level-set active contour segmentation for all slices that represents abdominal structure in the datasets.

Time efficiency is calculated based on equation (8) between semi-automatic and the proposed automatic segmentation for the level set active contour method:

$$
\text { Time efficiency }=\left(\frac{\text { TSAS }- \text { TAS }}{\text { TSAS }}\right) \times 100 \%
$$

where, TSAS is time required for semi-automatic segmentation which represent the user interactions and TAS is time required for automatic segmentation.

For the purpose of experimentation of processing time of semi-automatic segmentation, a subset of slices for each abdominal structure (five livers, spleen, right kidney and lift kidney) was selected using systematic random sampling. The random sampling is computed by selecting slices with an interval of 10 slices starting from the bottommost slice until topmost slice to ensure representation from all locations of an abdominal structure. For each of the slice, the computation time is referred to the time taken from the initialization of the contour curve in the slice until completion of the segmentation. Then the whole processing time of the dataset is calculated by taking an average time of the chosen subset slices, multiplied by the total number of slices in the whole abdominal structure. Whereas, the time of automatic segmentation (the proposed system) is calculated taking into 
account all the processes used in the proposed system including: SIFT feature extractions, finding closest slice, registration, rib bone extraction, initial slice segmentation, coronal view segmentation, multiple regions localization, and segmentation of all axial slices.

Table 7 represents the processing time obtained for both semi-automatic segmentation and automatic segmentation given by the proposed system. It is noticed that time efficiency by the proposed system increases with the increase of segmented slices being used. The time efficiency shown is an average of $68 \%$. The semi-automatic method takes longer processing time as compared to automatic system, because it requires manual initialization of contour curve in each slice of the dataset.

Table 7. Time performance of automatic and semi-automatic segmentations.

\begin{tabular}{lllll}
\hline Dataset name & Number of structure slices & Time of automatic segmentation [s] & Time of semi-automatic segmentation [s] & Time efficiency (\%) \\
\hline Liver1 & 102 & 5157 & 7935 & 35 \\
Liver3 & 57 & 1331 & 2665 & 50 \\
Liver4 & 140 & 5870 & 20210 & 71 \\
Liver5 & 163 & 9591 & 59546 & 84 \\
Liver6 & 73 & 989 & 3282 & 70 \\
Spleen & 74 & 1644 & 10205 & 84 \\
Left Kidney & 85 & 833 & 3230 & 74 \\
Right Kidney & 81 & 684 & 2608 & 73 \\
Average & & & & 68 \\
\hline
\end{tabular}

\section{Conclusion}

In this paper, the framework for knowledge guided medical image segmentation is presented. It offers flow to guide medical image segmentation active contour methods by managing between various sources of medical knowledge. And it could be the foundation of building a novel and flexible framework of segmenting medical images.

There are three key points that make this work is unique, which are: 1) automating and speeding active contour segmentation algorithms. 2) Involving and integrating between the semantic knowledge and image knowledge. 3) Minimizing the human interactions in medical image segmentation. It could be concluded based on the results of this study that the proposed method can handle the dissimilarity in intensity and shape between different datasets, produce better results in abdominal $\mathrm{CT}$ scans and minimize processing time of the semi-automatic segmentation method.

\section{References}

[1] Abdel-massieh, N. H., M. M. Hadhoud, et al. (2010). A fully automatic and efficient technique for liver segmentation from abdominal CT images. Informatics and Systems (INFOS), 2010 The 7th International Conference on.

[2] Campadelli, P., E. Casiraghi, et al. (2009). "Liver segmentation from computed tomography scans: A survey and a new algorithm." Artificial Intelligence in Medicine 45(2-3): 185-196.

[3] Casiraghi, E., P. Campadelli, et al. (2009). "Automatic Abdominal Organ Segmentation from CT images." Electronic Letters on Computer Vision and Image Analysis (ELCVIA) 8.

[4] Chan, T. F. and L. A. Vese (2001). "Active contours without edges." Image Processing, IEEE Transactions on 10(2): 266277.
[5] ChangYang, L., W. Xiuying, et al. (2010). Fully automated liver segmentation for low- and high- contrast CT volumes based on probabilistic atlases. 17th IEEE International Conference on Image Processing (ICIP).

[6] Chien-Cheng, L. and C. Pau-Choo (2000). Recognizing abdominal organs in CT images using contextual neural network and fuzzy rules. Engineering in Medicine and Biology Society, 2000. Proceedings of the 22nd Annual International Conference of the IEEE.

[7] Chunming, L., X. Chenyang, et al. (2005). Level set evolution without re-initialization: a new variational formulation. Computer Vision and Pattern Recognition, 2005. CVPR 2005. IEEE Computer Society Conference on.

[8] Cui, W., Y. Wang, et al. (2013). "Localized FCM Clustering with Spatial Information for Medical Image Segmentation and Bias Field Estimation." International Journal of Biomedical Imaging 2013: 8 .

[9] Ding, F., W. K. Leow, et al. (2005). Segmentation of 3D CT Volume Images Using a Single 2D Atlas. Computer Vision for Biomedical Image Applications, Springer Berlin / Heidelberg. 3765: 459-468.

[10] Foo, J. L. (2006). A Survey of User Interaction and Automation in Medical Image Segmentation Methods, Iowa State University.

[11] Furukawa, D., A. Shimizu, et al. (2007). Automatic liver segmentation method based on maximum a posterior probability estimation and level set method. 3D Segmentation In The Clinic: A Grand Challenge. T. H. a. M. S. a. B. v. Ginneken.

[12] Ginneken, B., T. Heimann, et al. (2007). 3D segmentation in the clinic: a grand challenge. 3D segmentation in the clinic: a grand challenge. B. Ginneken, T. Heimann and M. Stiner: 7-15.

[13] Ginneken, B. V., T. Heimann, et al. (2007). M.: 3D segmentation in the clinic: A grand challenge. 3D segmentation in the clinic: A grand Challenge2007.

[14] Hancock, E. R. and J. Kittler (1990). "Edge-labeling using dictionary-based relaxation." Pattern Analysis and Machine Intelligence, IEEE Transactions on 12(2): 165-181. 
[15] Haralick, R. (1973). Textural measures for images classification.

[16] Harms, J., M. Bartels, et al. (2005). "Computerized CT-Based 3D Visualization Technique in Living Related Liver Transplantation." Transplantation Proceedings 37(2): 10591062.

[17] Ibrahim, H., M. Petrou, et al. (2010). "Automatic Volumetric Liver Segmentation from MRI Data." International Journal of Computer Theory and Engineering (IJCTE) 2: 1793-8201.

[18] Jia, L. and J. Z. Wang (2008). "Real-Time Computerized Annotation of Pictures." Pattern Analysis and Machine Intelligence, IEEE Transactions on 30(6): 985-1002.

[19] Jiang, H., H. Tan, et al. (2013). "A Priori Knowledge and Probability Density Based Segmentation Method for Medical CT Image Sequences." BioMed Research International 2014: 11.

[20] Kallman, H.-E., E. Halsius, et al. (2008). "DICOM Metadata repository for technical information in digital medical images." Acta Oncologica 99999(1): 1-4.

[21] Kroon, D. J., E. v. Oort, et al. (2008). "Multiple Sclerosis Detection in Multispectral Magnetic Resonance Images with Principal Components Analysis." The MIDAS Journal.

[22] Lankton, S. and A. Tannenbaum (2008). "Localizing RegionBased Active Contours." Image Processing, IEEE Transactions on 17(11): 2029-2039.

[23] Lee, J., N. Kim, et al. (2007). Efficient Liver Segmentation exploiting Level-Set Speed Images with 2.5D Shape Propagation. 3D segmentation in the clinic: A grand Challenge2007: 189-196.

[24] Li, S., T. Fevens, et al. (2006). "Automatic clinical image segmentation using pathological modeling, PCA and SVM." Engineering Applications of Artificial Intelligence 19(4): 403410 .

[25] Linguraru, M. G., J. K. Sandberg, et al. (2010). Automated segmentation and quantification of liver and spleen from CT images using normalized probabilistic atlases and enhancement estimation. 37: 771-83.

[26] Liu, Y., T. Jiang, et al. (2005). Segmentation of 3D CT Volume Images Using a Single 2D Atlas. Computer Vision for Biomedical Image Applications, Springer Berlin / Heidelberg. 3765: 459-468.

[27] Lowe, D. G. (2004). "Distinctive Image Features from ScaleInvariant Keypoints." International Journal of Computer Vision 60(2): 91-110.

[28] Luo, S., X. Li, et al. (2014). "Review on the Methods of Automatic Liver Segmentation from Abdominal Images." Journal of Computer and Communications 2: 1-7.

[29] Martí, J., J. Benedí, et al. (2007). Automatic Segmentation of the Liver in CT Using Level Sets Without Edges. Pattern Recognition and Image Analysis, Springer Berlin / Heidelberg. 4477: 161-168.

[30] Mei, J., Y. Si, et al. (2013). "A novel active contour model for unsupervised low-key image segmentation." Central European Journal of Engineering 3(2): 267-275.

[31] MICCAI. (2007). "Workshop on 3D Segmentation in the Clinic - A Grand Challenge - Segmentation of the Liver", from http://mbi.dkfz-heidelberg.de/grand-challenge2007/.
[32] Möller, A. M. (2009). Challenges in Multimodality Imaging using Positron Emission Tomography, Technische Universität München.

[33] Nakayama, Y., Q. Li, et al. (2006). "Automated Hepatic Volumetry for Living Related Liver Transplantation At Multisection CT1." Radiology 240(3): 743-748.

[34] Narkhede, H. P. (2013). "Review of Image Segmentation Techniques." International Journal of Innovative Science and Modern Engineering 1(8): 54-61.

[35] O'Donnell, L. (2001). Semi-Automatic Medical Image Segmentation. Electrical Engineering and Computer Science, MASSACHUSETTS INSTITUTE OF TECHNOLOGY. Master.

[36] Pan, S. and B. Dawant (2001). Automatic 3D segmentation of the liver from abdominal CT images: a level-set approach. Medical Imaging 2001: Image Processing, SPIE.

[37] RadiologyAnatomyAtlas. (2010).

"http://web.mac.com/rlivingston/Site/Radiology_Anatomy_At las.html." from

http://web.mac.com/rlivingston/Site/Radiology_Anatomy_Atl as.html.

[38] Shin, H. S., B. H. Chung, et al. (2009). "Measurement of Kidney Volume with Multi-Detector Computed Tomography Scanning in Young Korean." Yonsei Med J 50(2): 262-265.

[39] SIG. (2010).

"http://sig.biostr.washington.edu/projects/AnnoteImage/." from http://sig.biostr.washington.edu/projects/AnnoteImage/.

[40] Slagmolen, P., A. Elen, et al. (2007). Atlas based liver segmentation using nonrigid registration with a b-spline transformation model. 3D Segmentation In The Clinic: A Grand Challenge. T. Heimann, M. Styner and B. v. Ginneken.

[41] Straka, M., A. L. Cruz, et al. (2004). Bone Segmentation in CT-Angiography Data Using a Probabilistic Atlas, Institute of Computer Graphics and Algorithms, Vienna University of Technology: 505--512.

[42] Tibamoso, G. and A. Rueda (2007). Semi-automatic Liver Segmentation From omputed Tomography (CT) Scans based on Deformable Surfaces 3D Segmentation In The Clinic: A Grand Challenge.

[43] Withey, D. J. and Z. J. Koles (2007). Medical Image Segmentation: Methods and Software. Noninvasive Functional Source Imaging of the Brain and Heart and the International Conference on Functional Biomedical Imaging, 2007. NFSI-ICFBI 2007. Joint Meeting of the 6th International Symposium on.

[44] Yasmin, M., M. Sharif, et al. (2013). "Neural networks in medical imaging applications: A survey." World Applied Sciences Journal 22(1): 85-96.

[45] Yongfu, H., J. Tianzi, et al. (2012). Iterative multi-atlas based segmentation with multi-channel image registration and Jackknife Context Model. Biomedical Imaging (ISBI), 2012 9th IEEE International Symposium on.

[46] Yuqian, Z., Z. Yunlong, et al. (2010). Fuzzy C-means clustering-based multilayer perceptron neural network for liver CT images automatic segmentation. Control and Decision Conference (CCDC), 2010 Chinese. 
[47] Zhou, N., T. Yang, et al. (2014). "An Improved FCM Medical Image Segmentation Algorithm Based on MMTD." Computational and Mathematical Methods in Medicine 2014: 8 .
[48] Zhou, W. and Y. Xie (2014). Interactive contour delineation and refinement in treatment planning of image-guided radiation therapy. 